East African Medical Journal Vol. 85 No. 3 March 2008

CONGENITAL LYMPHOEDEMA, BRONCHIECTASIS AND SEIZURE: CASE REPORT

S. Semiz, MD, Associate Professor, E. Dagdeviren, MD, Research Assistant and H. Ergin, MD, Professor, Department of Paediatrics, I. Kilic, MD, Associate Professor, S. Kirac MD, Professor, Department of Nuclear Medicine, M. Cimbis MD, Assistant Professor, Department of Paediatrics, and E. Semiz, MD, Professor, Pamukkale University School of Medicine, Department of Cardiology, Denizli, Turkey

Request for reprints to: Prof. S. Semiz, Camlaralti Mah. 6008 Sok. No: 1 Daire: 420070 Denizli-Turkey

\title{
CONGENITAL LYMPHOEDEMA, BRONCHIECTASIS AND SEIZURE: CASE REPORT
}

\author{
S. SEMIZ, E. DAGDEVIREN, H. ERGIN, I. KILIC, S. KIRAC, M. CIMBIS and E. SEMIZ
}

SUMMARY

\begin{abstract}
A 10-year-old girl with facial anomalies, mental retardation, peripheral lymphoedema, convulsions, cerebral cortical dysgenetic changes, bronchiectasis and chronic sinusitis is presented. She had features of both yellow nail syndrome and Hennekam syndrome. We think that our case might be a new congenital lymphoedema syndrome or an intermediate form between these syndromes.
\end{abstract}

\section{INTRODUCTION}

Mild degrees of congenital lymphoedema are considered common in the normal population, reflecting normal developmental variability in the regression of the lymphoedema present in every fetus before birth (1). The most common clinical causes of lymphedema are generally not inherited, occurring as results of trauma, surgery, neoplasia, infection or radiation. However, lymphoedema is due to a primary or an inherited cause in at least $10 \%$ of the cases (2). Hereditary lymphoedemas are developmental disorders of the lymphatics resulting in edema of the extremities due to altered lymphatic flow. Hereditary lymphoedema may occur as an isolated condition, examples of which include Milroy disease (OMIM 153100) and lymphoedema praecox (OMIM 153200), or as a component of a complex syndrome. Congenital lymphoedema is associated with several inherited complex syndromes including Hennekam syndrome and yellow nail syndrome (2). Hennekam syndrome is an autosomal recessive disorder comprising intestinal lymphangiectasia, lymphoedema, facial anomalies, and mental retardation (3). Yellow nail syndrome is characterised by yellow dystrophic nails in association with lymphedema and respiratory findings, including pleural effusion, bronchiectasis and chronic sinusitis (4). We present a 10-year-old girl with some characteristics of both syndromes, such as facial anomalies, mental retardation, peripheral lymphedema, convulsions, cerebral cortical dysgenetic changes, bronchiectasis and chronic sinusitis.

\section{CASE REPORT}

A 10-year-old girl presented with peripheral oedema, seizures and recurrent respiratory symptoms. Her infancy and early childhood were complicated by swelling of her limbs. She had frequently been admitted to various health centres because of recurrent pulmonary infections and convulsions. She had been hospitalised in another hospital a year ago due to pneumonia and pleural effusion before she was admitted to our hospital. She had grandmal seizures intermittently until a month ago. She had never been investigated because of convulsions, edemas and recurrent pulmonary infections.

She was born after an uncomplicated pregnancy via vaginal delivery. Physicomotor development was retarded. Her parents were first degree relatives. There was no family history of similar clinical features.

Her height and weight were $125 \mathrm{~cm}(<3 \mathrm{p})$ and $27 \mathrm{~kg}(10-25 \mathrm{p})$, respectively; the head circumference was $53 \mathrm{~cm}(50 \mathrm{p})$. A moderate mental retardation was present (I.Q.50). The patient had a short stature, an unusual face characterised by flat mid-face, and flat nasal bridge. Her upper and lower limbs were edematous (Figure 1). 
Figure 1

(a) Note the unusual face characterised by flat mid-face, and flat nasal bridge

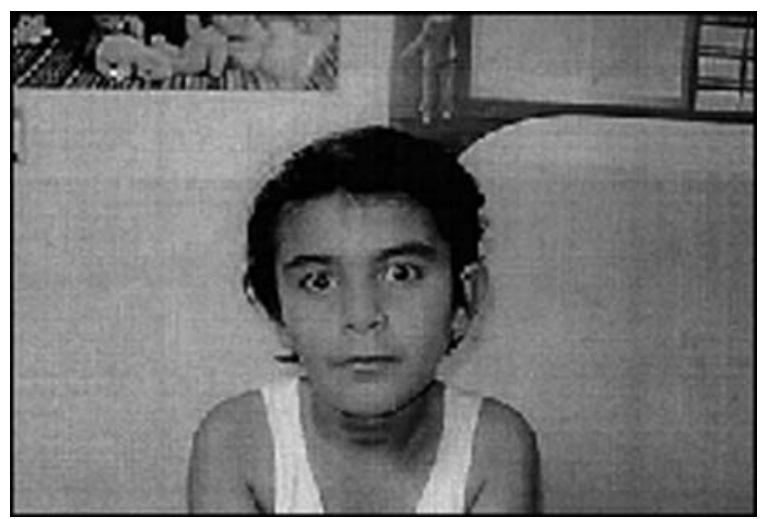

(b) Upper

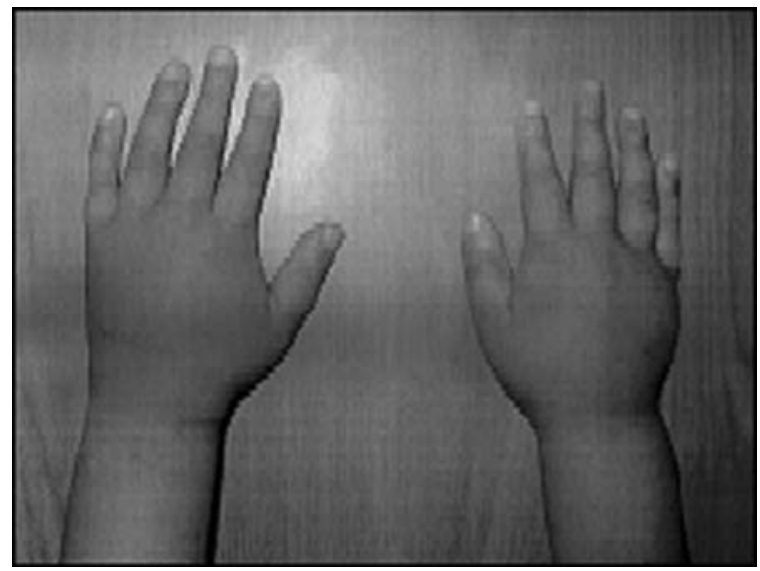

(c) lower limbs of the patient were edematous

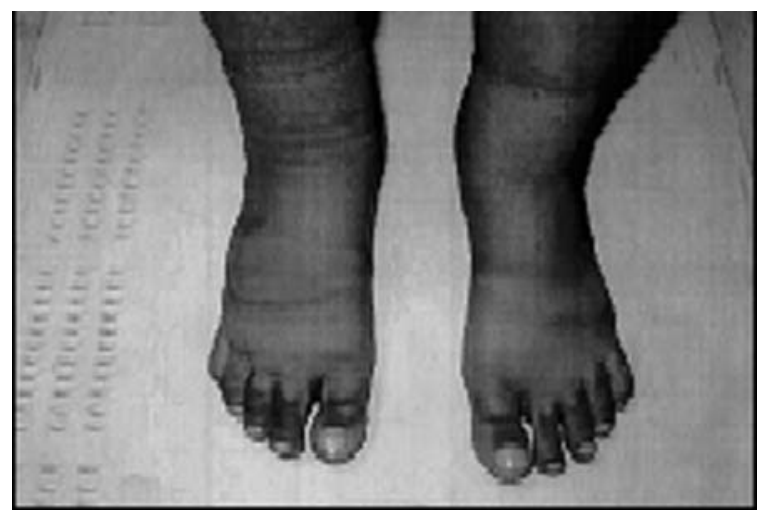

Complete blood count, serum protein levels, alpha-1 antitrypsin level, quantitative immunoglobulins, arterial blood gases, thyroid function tests, fecal lipid, fecal reducing substance, urine analysis, echocardiogram were all normal. Erythrocyte sedimentation rate was high and Creactive protein was positive. Her hearing test and eye examination were normal. Karyotype was normal. Both chest radiography and computed tomography of the thorax showed a widespread bilateral bronchiectasis. Pulmonary lymphangiectasia was not detected (Figure 2a). Sinus roentgenograms showed bilateral maxillary sinusitis. Cerebral magnetic resonance imaging (MRI) demonstrated macrogyria in both hemispheres (Figure 2b). Lymphoscintigraphy using $99 \mathrm{mTc}$ revealed no lymphatic drainage in both upper extremities and in the upper part of the right knee but the lymphatic drainage was normal in the left leg of the subject (Figure 3).

\section{Figure 2}

(a) Computed tomography of the thorax showed a widespread bilateral bronchiectasis

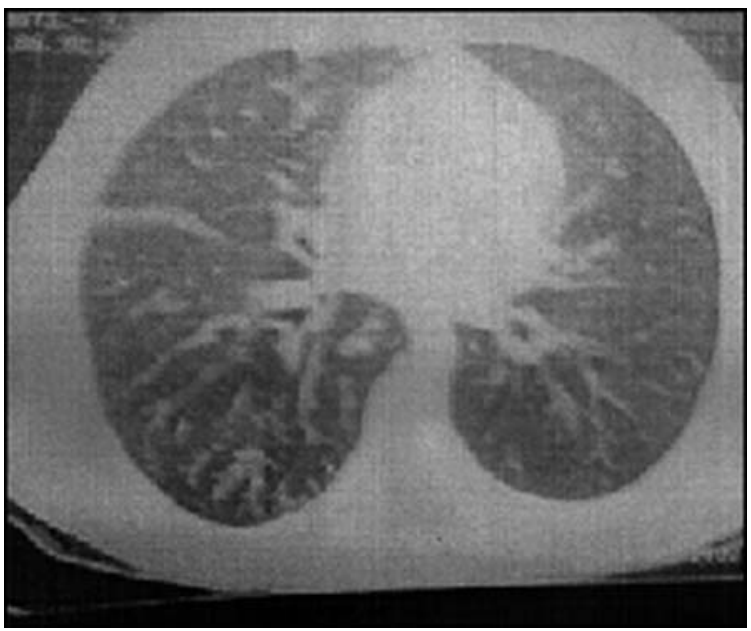

(b) Cerebral magnetic resonance imaging revealed macrogyria in both hemispheres

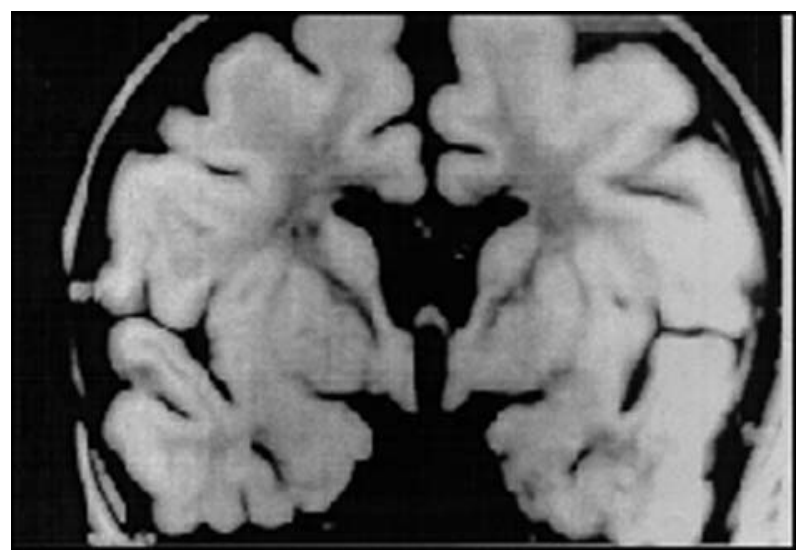


Figure 3

(a) Lymphoscintigraphy showed no lymphatic drainage in both upper extremities

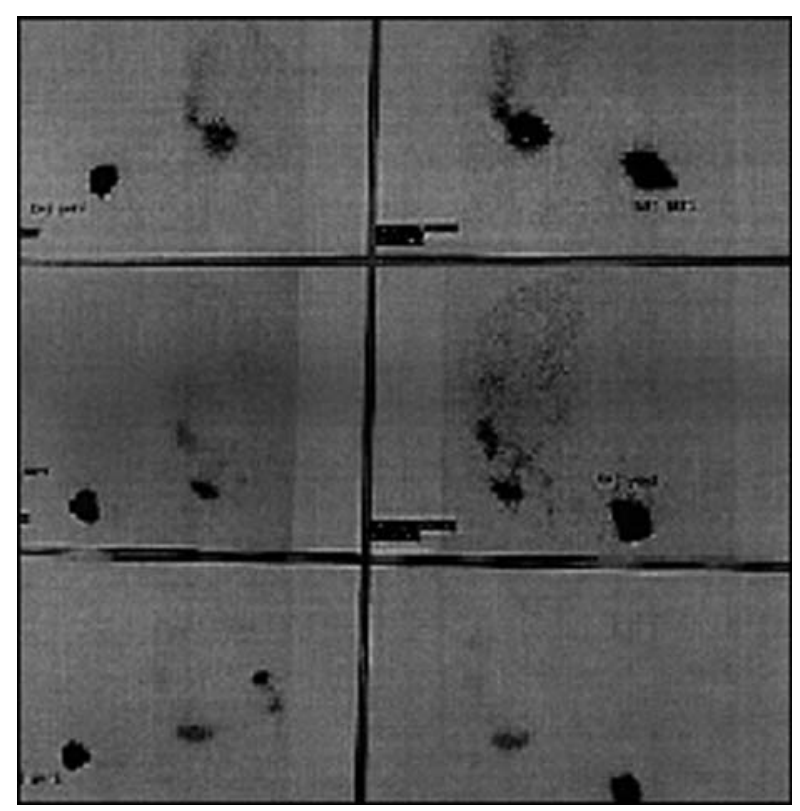

(b) and in the upper part of the right knee but the lymphatic drainage was normal in the left leg of the subject

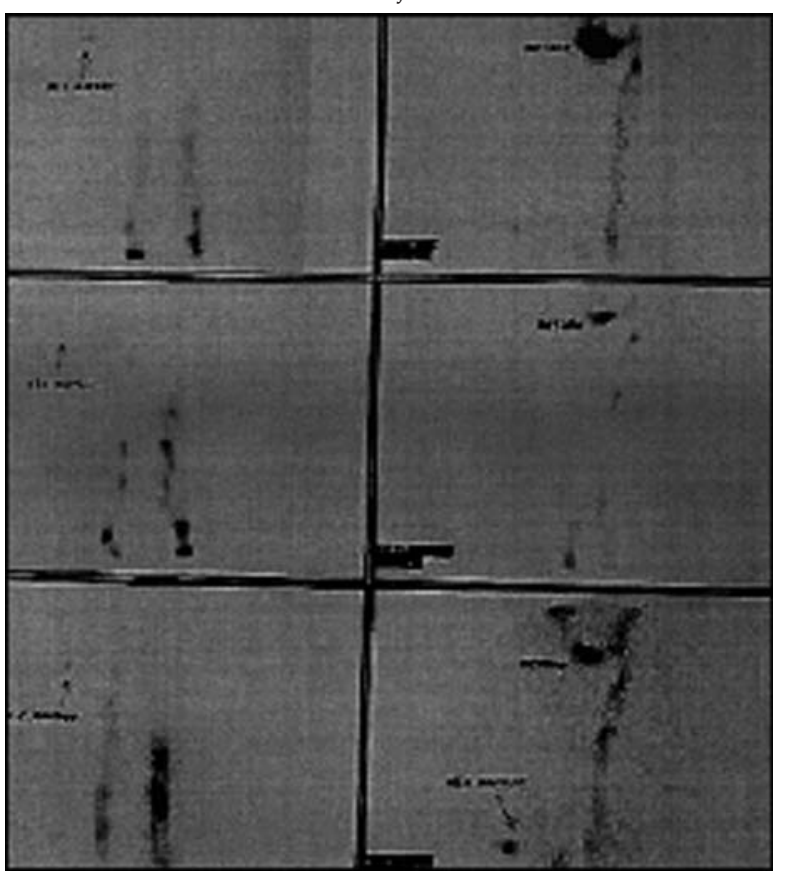

Electroencephalography showed a widespread epileptic activity. Renal ultrasound was normal. Intestinal biopsy was not performed, because she had no clinical signs of protein-losing enteropathy.

Antibiotics were given for bronchiectasis and sinusitis. Pneumonic symptoms of the subject persisted despite of postural drainage and antibiotherapy. Anticonvulsant treatment and a medium - chain triglyceride (MCT) - rich diet were begun.

\section{DISCUSSION}

In evaluating patients with possible Hennekam syndrome, most of the common causes of congenital lymphoedema can be discarded, either due to the presence of other symptoms or the absence of major symptoms of the present entity (3). Only a limited number of other syndromes can be difficult to differentiate from Hennekam syndrome. In 1995 Sailer et al (5) reported a case of Noonan syndrome associated with pulmonary lymphangiectasia and major lymphoedema of the lower extremities. Congenital lymphoedema may also be present in Turner syndrome. Our patient has not phenotypic findings of Turner or Noonan syndrome. Karyotype investigated for possible Turner syndrome was normal. In addition, pulmonary lymphangiectasia was not determined in our subject.

The phenotypic classification of dominantly inherited lymphoedema includes Milroy disease (OMIM153100), Meigelymphoedema (lymphoedema praecox) (OMIM 153200), lymphoedema-distichiasis syndrome (OMIM 153400), lymphoedema and ptosis (OMIM 153000 ) and yellow-nail syndrome (OMIM 153300). Milroy disease is asocciated with mutation in the FL T4 gene, whereas mutations in the FOXC2 gene were observed in the four lymphoedema syndromes that have phenotypic overlap (6). Lymphoedema distichiasis including yellow-nail syndrome caused by FOXC2 mutations (OMIM 602402) is characterised by onset of lymphoedema usually around puberty, lymphoedema predominantly in the lower limbs, hyperplasia of the lymphatics, and bronchiectasis. Milroy disease caused by VEGFR3 mutations (OMIM 136352) is characterised by onset of lymphoedema usually at birth, lymphoedema predominantly in the lower limbs, and hypoplasia of the lymphatics.

Yellow nail syndrome is characterised by yellow dystrophic nails in association with lymphedema and respiratory findings, including pleural effusion, bronchiectasis, and chronic sinusitis (4). Hiller et al (7) postulated that the cause of the pulmonary manifestations and lymphoedema was hypoplastic or deficient lymphatic vessels. Seventeen patients with yellow nail syndrome were evaluated by Varney et al (8). Fourteen of the patients suffered from severe rhinosinusitis, in which pre-dated nail changes were seen in four subjects, coincided with yellow nails in six, and occurred later in the rest. The age at onset of symptoms is variable, ranging from birth to the eight decade. Lymphoedema is usually the first clinical manifestation, but in some cases nail findings appear the first (4). Over 100 cases have been published, most of which have been sporadic (9). All of the characteristics, except typical dystrophic nail changes, of yellow - nail syndrome were present in our subject but there was no family history of similar 
clinical features. Dystrophic nail changes might occur at follow-up. However, though convulsions had not been described in this syndrome, our subject had seizures and cerebral cortical changes.

Hennekam et al. (3) described a syndrome of intestinal lymphangiectasia; severe lymphoedema of limbs, genitalia, and face; facial anomalies (flat mid-face, flat nasal bridge, hypertelorism, small mouth, tooth anomalies, and small ears), seizures, mild growth retardation and moderate mental retardation. Autosomal recessive inheritance was strongly supported by the occurrence of the disorder in two males and two females of two sibships from parents who shared a common ancestral couple. Van Balkom et al (10) reported eight patients, and compared their findings to 16 cases in the literature. In addition to these 24 cases, eight more subjects with Hennekam syndrome have been reported by different authors in the literature (11-15). The lymphoedema was usually congenital and, often, gradually progressive. The lymphangiectasias were present in the intestines, but were also found in the pleura, pericardium, thyroid gland, and kidney. Several patients demonstrated congenital cardiac and blood vessel anomalies, pointing to a disturbance of angiogenesis in at least some of the patients. Facial features were variable, and were chiefly characterised, in a typical patient, by a flat face, flat and broad nasal bridge, and hypertelorism. Other anomalies included glaucoma, dental anomalies, hearing loss, and renal anomalies. Convulsions were common. In addition, Huppkeet al. (16), using MRI, defined several cerebral abnormalities including small subcortical hyperintensities, a large cystic lesion and pachygyria in the parietal place of two cases. There were findings including lymphoedema of limbs, facial anomalies, seizures, growth retardation, and moderate mental retardation, macrogyria in our subject, that were all the characteristics of Hennekam syndrome. The status that her parents were first degree relatives supports autosomal recessive Hennekam syndrome. On the contrary, our patient had no pulmonary and intestinal lymphangiectasias, which are the major findings of Hennekam syndrome. However, intestinal lymphangiectasia has been present in most (but not all) patients. For this reason, the patient should probably be diagnosed as Hennekam syndrome, with bronchiectasis and chronic sinusitis as additional findings.

The constellation of the clinical manifestations in the present patient is not compatible with above any clinic entity. Thus, it may be crucial to investigate chromosomal microdeletion at 16q24.3 region around FOXC2. Aetiology of the congenital lymphoedema syndromes has not been understood completely yet. Bull et al. (17) suggested that the underlying cause of yellow nail syndrome is not primarily a lymphatic abnormality and the lymphatic impairment associated with yellow nail syndrome appears to be secondary, and predominantly functional in nature, rather than due to structural changes.

There is not a specific treatment in congenital lymphoedema syndromes. However, diet is very important in treatment. Conservative management, including a low fat intake supplemented with MCT which are absorbed directly into the portal venous system without entry into the lymphatic system, has been suggested in children with cyclothorax $(18,19)$. We initiated the same diet, including a low fat intake supplemented with MCT, considering thehypoplastic lymphatic vessels to be the possible eatiopathogenesis of this lymphoedema syndrome. There has been a moderate improvement in oedema of the lower extremities.

In conclusion, our subject has some features of both yellow nail syndrome and Hennekam syndrome. This type of presentation has not been reported in the literature yet. We think that our case is a new congenital lymphoedema syndrome or an intermediate form between these syndromes.

\section{REFERENCES}

1. Optiz J.M. Editorial comment on the paper by Crowe and Dickerman: on congenital lymphedema. Am. J. Med. Genet. 1986; 24: 127-129.

2. Allanson J. E. Lymphatic circulation. In: Stevenson R.E., Hall J.G., Goodman R.M., (eds). Human malformations and related anomalies. Oxford University Press, New York, 1993; 271-307.

3. Hennekam R.C.M., Geerdink R.A., Hamel B.C., et al. Autosomal recessive intestinal lymphangiectasia and lymphedema, with facial anomalies and mental retardation. Am. J. Med. Genet. 1989; 34: 593-600.

4. Eastwood H.D. and Williams T. J. Pleural effusions and yellow nails of late onset. Postgrad. Med. J. 1973; 49: 364-365.

5. Sailer M., Unsinn K., Fink C., Covi B. and Gassner I. Pulmonary lymphangiectasis with spontaneous chylothorax in Noonan syndrome. Klin. Padiatr. 1995; 207: 302-304.

6. Finegold D.N., Kimak M.A., Lawrence E.C., et al. Truncating mutations in FOXC2 cause multiple lymphedema syndromes. Hum. Molec. Genet. 2001; 10: 1185-1189.

7. Hiller E., Rosenow E.C. and Olsen A.M. Pulmonary manifestations of the yellow nail syndrome. Chest. 1978; 61: 452-458.

8. Varney V. A., Cumberworth V., Sudderick R., Durham S.R. and Mackay I. S. Rhinitis, sinusitis and the yellow nail syndrome: a review of symptoms and response to treatment in 17 patients. Clin. Otolaryngol. 1994; 19: 237-240.

9. Hogue S.R., Mansour S. and Mortimer P.S. Yellow nail syndrome: not a genetic disorder? Eleven new cases and a review of the literature. Br. J. Dermatol. 2007; 156: 1230-1234. 
10. Van Balkom I.D.C., Alders M., Allanson J., et al. Lymphedema-lymphangiectasia-mental retardation (Hennekam) syndrome: a review. Am. J. Med. Genet. 2002; 112: 412-421.

11. Bellini C., Mazzella M., Arioni C., et al.Hennekam syndrome presenting as nonimmune hydrops fetalis, congenital chylothorax, and congenital pulmonary lymphangiectasia. Am. J. Med. Genet. A 2003; 120: 92-96.

12. AI-Gazali L.I., Hertecant J., Ahmed R., Khan N.A., and Padmanabhan R. Further delineation of Hennekam syndrome. Clin. Dysmorphol. 2003; 12: 227-232.

13 Guardiano M., Lobo A., Nunes T. and Vaz L. Recurrent chylothorax - a fifteen years long history. Rev. Port. Pneumol. 2004; 10: 253-258.

14. Heruth M., Muller P., Liebscher L. et al. Exudative enteropathy in congenital lymphedemalymphangiectasia syndrome. Klin. Padiatr. 2006; 218: 27-30.
15. Musumeci M.L., Nasca M.R., De Pasquale R. et al. Cutaneous manifestations and massive genital involvement in Hennekam syndrome. Pediatr. Dermatol. 2006; 23: 239-242.

16. Huppke P., Christen H.J., Sattler B. and Hanefeld F. Two broters with Hennekam syndrome and cerebral abnormalities. Clin. Dysmorphol. 2000; 9: 21-24.

17. Bull R.H., Fenton D.A. and Mortimer P.S. Lymphatic function in the yellow nail syndrome. Br.J. Dermatol. 1996; 134: 307-312.

18 Jalili F. Medium-chain triglycerides and total parenteral nutrition in the management of infants with congenital chylothorax. South Med. J. 1987; 80: 1290-1293.

19. Densupsoontorn N.S., Jirapinyo P., Wongarn R., et al. Management of chylothorax and chylopericardium in pediatric patients: experiences at Siriraj Hospital, Bangkok. Asia Pac. Clin. Nutr. 2005; 14: 182-187. 